\title{
A CHARACTERIZATION OF OSTERWALDER-SCHRADER PATH SPACES BY THE ASSOCIATED SEMIGROUP
}

\author{
BY ABEL KLEIN
}

Communicated by Alexandra Bellow, May 7, 1976

This note is to announce a characterization of (generalized) path spaces satisfying the Osterwalder-Schrader positivity condition by the associated semigroup, on the lines of the characterization of Markov path spaces by positivity preserving semigroups (e.g. Simon [5], Klein and Landau [3]). In the semigroup characterization Osterwalder-Schrader path spaces are seen to be the natural generalization of Markov path spaces. As an application we discuss the existence of Euclidean fields given a relativistic Wightman field theory.

I. Path spaces and semigroups. A (generalized) path space $\left((Q, \Sigma, \mu), \Sigma_{0}\right.$, $U(t), R)$ consists of a probability space $(Q, \Sigma, \mu)$; a distinguished sub- $\sigma$-algebra $\Sigma_{0}$; a one-parameter group $U(t)$ of measure preserving automorphisms of $L_{\infty}(Q, \Sigma, \mu)$ which are strongly continuous in measure; a measure preserving automorphism $R$ of $L_{\infty}(Q, \Sigma, \mu)$ such that $R^{2}=I, R U(t)=U(-t) R$, and $R E_{0}$ $=E_{0} R$ where $E_{0}$ is the conditional expectation with respect to $\Sigma_{0}$; where $\Sigma$ is generated by $\bigcup_{t \in R} \Sigma_{t}, \Sigma_{t}=U(t) \Sigma_{0}$. By $E_{+}\left(E_{-}\right)$we will denote the conditional expectation with respect to $\Sigma_{+}\left(\Sigma_{-}\right)$, the $\sigma$-algebra generated by $\bigcup_{t \geqslant 0} \Sigma_{t}$ $\left(\bigcup_{t \leqslant 0} \Sigma_{t}\right)$. The path space is said to be Osterwalder-Schrader if $\langle R F, F\rangle \geqslant 0$ for every $F \in L_{2}\left(Q, \Sigma_{+}, \mu\right)$. It is said to be Markov if $R E_{0}=E_{0}$ and $E_{+} E_{-}=$ $E_{+} E_{0} E_{-}$.

Every Markov path space is Osterwalder-Schrader [4]. In the case of a Markov path space $P(t)=E_{0} U(t) E_{0}$ gives a positivity preserving semigroup on $L_{2}\left(Q, \Sigma_{0}, \mu\right)[5],[3]$. Given an Osterwalder-Schrader path space there exists [4] a Hilbert space $H$ and a contraction $V: L_{2}\left(Q, \Sigma_{+}, \mu\right) \rightarrow H$ such that $V$ has dense range and $P(t) V(F)=V(U(t) F)$ for $F \in L_{2}\left(Q, \Sigma_{+}, \mu\right)$ and $t \geqslant 0$ defines a strongly continuous selfadjoint contraction semigroup on $H$. If $\Omega=V(1)$, then $\|\Omega\|=1$ and $P(t) \Omega=\Omega$ for all $t \geqslant 0$.

For Osterwalder-Schrader path spaces we must look at another piece of structure, which is hidden in the Markov case.

LemmA. Let $\left((Q, \Sigma, \mu), \Sigma_{0}, U(t), R\right)$ be an Osterwalder-Schrader path

AMS (MOS) subject classifications (1970). Primary 60J99, 81A17, 81A18; Secondary 47D05, 60G20.

Key words and phrases. Path spaces, Osterwalder-Schrader positivity, Markov property, positive semigroup structure. 
space, and let $H, V, P(t), \Omega$ be as above. Then, if $f \in L_{\infty}\left(Q, \Sigma_{0}, \mu\right), \widetilde{f} \cup(F)=$ $V(f F)$ for $F \in L_{2}\left(Q, \Sigma_{+}, \mu\right)$ defines a bounded operator on $H$ with $\|\tilde{f}\|=$ $\|f\|_{\infty}$, and $\mathfrak{Q}=\left\{\widetilde{f} \mid f \in L_{\infty}\left(Q, \Sigma_{0}, \mu\right)\right\}$ is a commutative von Neumann algebra of operators on $\mathrm{H}$, with $\Omega$ as a separating vector. Moreover, for any $t_{1} \leqslant t_{2} \leqslant$ $\cdots \leqslant t_{n}, f_{t_{i}}=U\left(t_{i}\right) f_{i}$ where $f_{i} \in L_{\infty}\left(Q, \Sigma_{0}, \mu\right)$ and $i=1,2, \ldots, n$,

$$
\int f_{t_{1}} f_{t_{2}} \cdots f_{t_{n}} d \mu=\left\langle\Omega, \tilde{f}_{1} P\left(t_{2}-t_{1}\right) \tilde{f}_{2} \cdots P\left(t_{n}-t_{n-1}\right) \tilde{f}_{n} \Omega\right\rangle .
$$

$(H, P(t), \mathfrak{A}, \Omega)$ is called the associated semigroup structure. If $\left(\left(Q, \Sigma_{0}, \mu\right)\right.$, $\left.\Sigma_{0}, U(t), R\right)$ is a Markov path space, $\left(L_{2}\left(Q, \Sigma_{0}, \mu\right), E_{0} U(t) E_{0}, L_{\infty}\left(Q, \Sigma_{0}, \mu\right), 1\right)$ is its associated semigroup structure.

Definition. A positive semigroup structure $(H, P(t), \mathfrak{A}, \Omega)$ consists of a Hilbert space $H$; a strongly continuous selfadjoint contraction semigroup $P(t)$ on $\mathrm{H}$; a commutative von Neumann algebra $\mathscr{U}$ of operators on $\boldsymbol{H}$; a unit vector $\Omega \in H$; such that $P(t) \Omega=\Omega$ for all $t \geqslant 0 ; \Omega$ is a cyclic vector for the algebra generated by $\mathfrak{A} \cup\{P(t) \mid t \geqslant 0\}$, i.e. the linear span of $\left\{P\left(t_{1}\right) f_{1} P\left(t_{2}\right) \ldots\right.$ $\left.P\left(t_{n}\right) f_{n} \Omega \mid f_{1}, \ldots, f_{n} \in \mathscr{A}, t_{1}, \ldots, t_{n} \geqslant 0\right\}$ is dense in $H$; and for all $f_{1}, \ldots$, $f_{n} \in \mathfrak{U}^{+}=\{f \in \mathscr{U} \mid f \geqslant 0\}$ and $t_{1}, \ldots, t_{n} \geqslant 0,\left\langle\Omega, P\left(t_{1}\right) f_{1} P\left(t_{2}\right) \cdots\right.$ $\left.P\left(t_{n}\right) f_{n} \Omega\right\rangle \geqslant 0$.

Osterwalder-Schrader path spaces are characterized by positive semigroup structures.

TheOREM. Let $\left((Q, \Sigma, \mu), \Sigma_{0}, U(t), R\right)$ be an Osterwalder-Schrader path space and $(H, P(t), \mathscr{U}, \Omega)$ its associated semigroup structure. Then $(H, P(t), \mathfrak{A}$, $\Omega)$ forms a positive semigroup structure.

Conversely, let $(H, P(t), \mathfrak{A}, \Omega)$ be a positive semigroup structure. Then there exists an Osterwalder-Schrader path space such that $(H, P(t), \mathfrak{U}, \Omega)$ is its associated semigroup structure.

COROllary. Let $\left((Q, \Sigma, \mu), \Sigma_{0}, U(t), R\right)$ be an Osterwalder-Schrader path space, and $(H, P(t), \mathfrak{U}, \Omega)$ its associated semigroup structure. The path space is Markov if and only if $\Omega$ is a cyclic vector for $\mathfrak{Q}$.

The details will appear elsewhere [2].

II. Existence of Euclidean fields. Our Theorem can be used to construct Euclidean fields given a relativistic Wightman field theory, in the same way Simon ([5], [6, Chapter IV]) used the similar result for Markov path spaces and positivity preserving semigroups to construct Euclidean fields. In Simon's scheme Axioms (S3) and (S4) [6, p. 120] are the basic elements in the construction of Euclidean fields. We can replace these axioms by the weaker:

AxIom 3'. The von Neumann algebra $\mathfrak{Q}$ generated by the time zero fields is abelian; and the vacuum $\Omega$ is a cyclic vector for the von Neumann algebra generated by the fields at all fixed times. 
Axıom $4^{\prime}$. For all $F_{1}, \ldots, F_{n} \in \mathfrak{Q}^{+}=\{F \in \mathfrak{U} \mid F \geqslant 0\}$ and $t_{1}, \ldots, t_{n}$ $\geqslant 0,\left\langle\Omega, e^{-t_{1} H} F_{1} e^{-t_{2} H} F_{2} \cdots e^{-t_{n} H} F_{n} \Omega\right\rangle \geqslant 0$.

We can then construct Euclidean fields satisfying Nelson's axioms, except for the Markov property which is replaced by the Osterwalder-Schrader positivity condition.

A detailed version of our axiom scheme will appear elsewhere [1], [2].

\section{REFERENCES}

1. A. Klein, When do Euclidean fields exist?, Letters in Mathematical Physics, 1 (1976), 131-133.

2. The semigroup characterization of Osterwalder-Schrader path spaces and the construction of Euclidean fields (to appear).

3. A. Klein and L. Landau, Singular perturbations of positivity preserving semigroups via path space techniques, J. Functional Anal. 20 (1975), 44-82.

4. K. Osterwalder and R. Schrader, Axioms for Euclidean Green's functions. I, II, Comm. Math. Phys. 31 (1973), 83-112; ibid. 42 (1975), 281-305.

5. B. Simon, Positivity of the Hamiltonian semigroup and the construction of Euclidean region fields, Helv. Phys. Acta 46 (1972), 686-696.

6. - The $P(\phi)_{2}$ Euclidean (quantum) field theory, Princeton Series in Physics, Princeton Univ. Press, Princeton, N. J., 1974.

DEPARTMENT OF MATHEMATICS, UNIVERSITY OF CALIFORNIA, IRVINE, CALIFORNIA 92717 